\title{
Bordat, Josef
}

\section{Menschenrechtskrieg und Menschenrechtserziehung}

Bildungsforschung 3 (2006) 1, 22 S.

Quellenangabe/ Reference:

Bordat, Josef: Menschenrechtskrieg und Menschenrechtserziehung - In: Bildungsforschung 3 (2006) 1, 22 S. - URN: urn:nbn:de:0111-opus-46478 - DOI: 10.25656/01:4647

in Kooperation mit / in cooperation with:

\section{bildungsforschung}

http://www.bildungsforschung.org

\section{Nutzungsbedingungen}

Gewährt wird ein nicht exklusives, nicht übertragbares, persönliches und beschränktes Recht auf Nutzung dieses Dokuments. Dieses Dokument ist ausschließlich für den persönlichen, nicht-kommerziellen Gebrauch bestimmt. Die Nutzung stellt keine Übertragung des Eigentumsrechts an diesem Dokument dar und gilt vorbehaltlich der folgenden Einschränkungen: Auf sämtlichen Kopien dieses Dokuments müssen alle Urheberrechtshinweise und sonstigen Hinweise auf gesetzlichen Schutz beibehalten werden. Sie dürfen dieses Dokument nicht in irgendeiner Weise abändern, noch dürfen Sie dieses Dokument für öffentliche oder kommerzielle Zwecke vervielfältigen, öffentlich ausstellen, aufführen, vertreiben oder anderweitig nutzen.

Mit der Verwendung dieses Dokuments erkennen Sie die Nutzungsbedingungen an.

\section{Terms of use}

We grant a non-exclusive, non-transferable, individual and limited right to using this document.

This document is solely intended for your personal, non-commercial use. Use of this document does not include any transfer of property rights and it is conditional to the following limitations: All of the copies of this documents must retain all copyright information and other information regarding legal protection. You are not allowed to alter this document in any way, to copy it for public or commercial purposes, to exhibit the document in public, to perform, distribute or otherwise use the document in public.

By using this particular document, you accept the above-stated conditions of use.

\section{Kontakt / Contact:}

\section{peDOCS}

DIPF | Leibniz-Institut für Bildungsforschung und Bildungsinformation Informationszentrum (IZ) Bildung

E-Mail: pedocs@dipf.de

Internet: www.pedocs.de

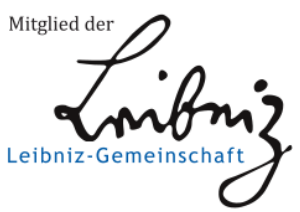




\title{
Menschenrechtskrieg und Menschenrechtserziehung
}

\author{
Josef Bordat
}

Reaktion und Prävention - unter diesen Schlagwörtern wird momentan der richtige Umgang der Weltgemeinschaft mit kriegerischer Gewalt und Terrorismus gesucht. Zum einen versucht der humanitäre Interventionismus durch "Menschenrechtskriege“ den Schutz der Menschenrechte zu gewährleisten, zum anderen unternimmt die UNO vermehrt Anstrengungen, durch „Menschenrechtsbildung“ präventiv tätig zu werden. Der diesbezügliche Diskussionsstand wird unter besonderer Berücksichtigung des Gutachtens The Responsibility To Protect (2001) der International Commission on Intervention and State Sovereignty und der Aktivitäten und Pläne der Vereinten Nationen zur Menschenrechtsbildung dargestellt.

\section{Einleitung}

Nach dem Zusammenbruch der bipolaren Weltordnung hat sich der Begriff des Krieges gewandelt. Krieg ist nicht mehr nur die gewaltsame Auseinandersetzung zweier oder mehrerer souveräner Staaten, die ihre „unterschiedlichen souveränen Willen“ (Hegel 1986 [1821], 497 [§ 330]) zum Ausdruck bringen und denen der Krieg, wenn „die besonderen Willen keine Übereinkunft finden“ (Hegel 1986 [1821], 500 [§ 333]), als Mittel zur Durchsetzung von Interessen dient [1], sondern auch und vor allem jene Gewalt, die von nicht-staatlichen Gruppen (Bürgerkriegsparteien, Clans, Terroristen) bzw. gegen diese („War on terror“, humanitärer Interventionismus) ausgeübt wird. Das Ende der Konfrontation ideologischer Blöcke hat ethnische und religiöse Spannungen ausbrechen lassen, die schon lange unter der Oberfläche der Bündnistreue zur jeweiligen Hegemonialmacht schwelten. Die grausamen (Bürger-)Kriege auf dem Balkan, aber auch die nach wie vor ungelösten Konflikte in den jungen Kaukasusrepubliken haben in den vergangenen 15 Jahren gezeigt, dass das Ende der Sowjetunion die Wahrscheinlichkeit von Kriegen in Europa und vor den Toren Europas nicht herabgesenkt, sondern dramatisch gesteigert hat. Hinzu kommen gewaltsame ethnische Konflikte in Afrika, die zum einen von gestiegenem ökonomischem Interesse an wichtigen Rostoffen, zum anderen von nachlassenden politischen 
Ausgabe 2006

Jahrgang 3 Ausgabe 1

Schwerpunkt "Krieg und

Bildung" (hrsg. von

Elisabeth Zwick)

ISSN 1860-8213

\section{bildungsforschung \\ http://bildungsforschung.org}

Bindungen an die ehemaligen Kolonial- und späteren Schutzmächte begünstigt werden; das Massaker in Ruanda (1994) ist dazu nur ein besonders eindrückliches Beispiel. Schließlich ist auch der Terrorismus im Nahen und Mittleren Osten ein Ausdruck neuer kriegerischer Entfaltungsmöglichkeiten, die durch das Machtvakuum hervorgerufen werden, welches das SowjetImperium hinterlassen hat.

Mehr denn je scheint durch die asymmetrische Bedrohung und die alltägliche Gewalt schließlich die Gewährleistung der Menschenrechte in Gefahr. Immer stärker rücken die Menschenrechte in den Blickpunkt des Interesses, sei es, dass ihre Verletzung als Richtschnur für die Bewertung von Konflikten oder aber ihr Schutz als Begründung für ein Eingreifen in eben jene Konflikte gilt.

Diesem Wandel des Kriegsbegriffs entspricht der Versuch einer Reform des Völkerrechts bzw. einer Anpassung der UNO-Politik: Das Gewaltverbot und das Nichteinmischungsgebot der UN-Charta (Art. 2 Zf. 4 sowie Art. 2 Zf. 7), die noch den alten Kriegsbegriff unterstellt, soll einem Interventionsrecht weichen, wie es die International Commission on Intervention and State Sovereignty (ICISS) vorschlägt. Gleichzeitig sollen die Menschenrechte dadurch gestärkt werden, dass Bildung und Erziehung die Menschenrechte in den Vordergrund rücken, getragen vom Gedanken eines „Menschenrechts auf Menschenrechtsbildung“ zur Verwirklichung einer „Kultur der Menschenrechte". Beiden Spuren möchte ich im folgenden Aufsatz nachgehen.

\section{Menschenrechtskriege}

\subsection{Klärungsbedarf}

Mit dem Zusammenbruch der bipolaren Weltordnung bekam zugleich auch die alte Völkerrechtsordnung Risse, deren Säulen Souveränität, Territorialität und Nichteinmischung sich im beginnenden Zeitalter der Globalisierung rasch als nicht tragfähig erweisen sollten. Endgültig zusammengebrochen ist diese Ordnung am 11. September 2001, begraben unter den Trümmern des World Trade Center. Zwischen diesen Ereignissen lag der Kosovo-Krieg (1999), der eine wahre Flut an Stellungnahmen prominenter Denker ausgelöst hat, die sich der NATO-Lage zwischen „Macht und Moral“ (Bald 1999) annahmen und damit zur Verabschiedung des alten Krieges anstimmten. So äußerte sich etwa Jürgen Habermas zum Dilemma des Westens angesichts des Gräuels und verteidigte den NATO-Angriff, was ihm Unverständnis eintrug und ihn schließlich sogar zum „Hegel der Bundesrepublik“ (Ross 2001) mutieren ließ. 
Ausgabe 2006

Jahrgang 3 Ausgabe 1

Schwerpunkt "Krieg und

Bildung" (hrsg. von

Elisabeth Zwick)

ISSN 1860-8213

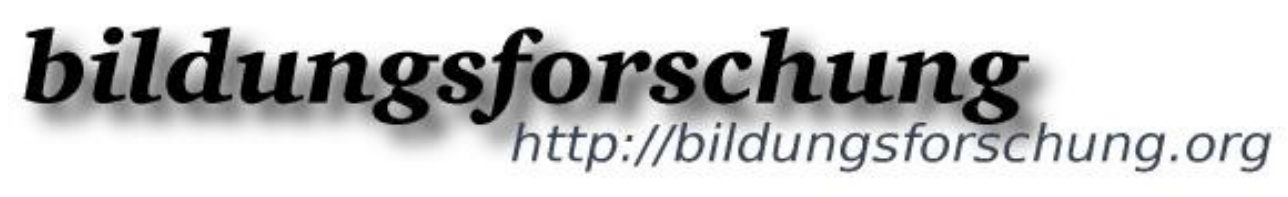

Auch Ulrich Beck hatte sich für den NATO-Einsatz ausgesprochen. Er erklärte das Zeitalter des clausewitz'schen Krieges für beendet, da im Zeitalter der postnationalen Intervention die klassische Form des 19. und 20. Jahrhunderts, Kriege mit Hilfe von Interesse, Rivalität und Feindschaft der Staaten zu legitimieren, vorüber sei. Nach Clausewitz gibt es Krieg, wenn die Diplomatie scheitert, nach Beck geht es um eine „neue Ethik globaler Demokratie und Menschenrechte“, die den Übergang zweier Zeitalter symbolisiere, „von einer nationalstaatlichen zu einer kosmopolitischen Weltordnung " (Beck 1999, 985 f.). Der Krieg im Kosovo sei da nur der Anfang einer neuen Form der Kriegsführung, die mit neuen Maßnahmen völkerrechtlicher Art korrespondieren muss, die wiederum auf einem neuen Verständnis von Staat und Souveränität basieren.

Bei allen skeptischen Vorbehalten gegen einen „Weltstaat" und eine „Weltregierung“ wird derzeit über eine verbindliche Pflicht der Weltgemeinschaft, über den Sicherheitsrat klare Kriterien für Gründe, Verlauf, Ziel und Nachbereitung einer Intervention zu erarbeiten und in geeignete Rechtsform $\mathrm{zu}$ bringen, vehement diskutiert. Interventionen in Fällen von ethnischen Konflikten, Bürgerkrieg und Gewaltakten in genozidaler Absicht, brauchen eine Rechtsgrundlage, welche die willkürliche Kasuistik ersetzt, die momentan die Entscheidungskultur des UN-Sicherheitsrats prägt. Ein Vorschlag aus dem Jahre 2001, das Gutachten The Responsibility To Protect der International Commission on Intervention and State Sovereignty (ICISS), trägt dem Rechnung.

Gleich zu Beginn des Gutachtens The Responsibility To Protect der kanadischen Regierung vom Dezember 2001, das von der International Commission on Intervention and State Sovereignty (ICISS) [2] erarbeitet und vom International Development Research Centre (IDRC) herausgegeben wurde, zeigt sich sowohl der Bezug zum aktuellen Schrecken des Terrors, als auch zugleich die abwägend-distanzierte Grundhaltung, die den ganzen Bericht prägt: „This report is about the so-called ,right of humanitarian intervention': the question of when, if ever, it is appropriate for states to take coercive - and in particular military - action, against another state for the purpose of protecting people at risk in that other state. At least until the horrifying events of 11 September 2001 brought to center stage the international response to terrorism, the issue of intervention for human protection purposes has been seen as one of the most controversial and difficult of all international relations questions." (IDRC 2001, VII). Es folgt eine weitere historische Motivierung und die Konkretisierung der Aufgabenstellung: „With the end of the Cold War, it became a live issue as never before. Many calls for intervention have been made over the last decade 
Ausgabe 2006

Jahrgang 3 Ausgabe 1

Schwerpunkt "Krieg und

Bildung" (hrsg. von

Elisabeth Zwick)

ISSN 1860-8213

\section{bildungsforschung \\ http://bildungsforschung.org}

- some of them answered and some of them ignored. But there continues to be disagreement as to whether, if there is a right of intervention, how and when it should be exercised, and under whose authority." (ebd.). Die Frage lautet also: Sind militärische Interventionen erlaubt, und wenn ja: wann, wie und unter wessen Führung? Damit wird in Kontinuität des traditionellen bellum iustum-Topos vorgegangen, indem zunächst die Frage des ius ad bellum und dann die des ius in bello adressiert wird.

Zur Beantwortung der Frage, ob es einen „gerechten Krieg“ geben kann und wenn ja -, an welche Bedingungen die "Gerechtigkeit" dieses Krieges geknüpft ist, bezieht sich die aktuelle Debatte auf die Geschichte des europäischen Mittelalters bzw. der frühen Neuzeit. In diesem Sinne möchte ich die patristisch-scholastische Tradition, also namentlich Augustinus und Thomas von Aquin, heranziehen, die auch dem Diskurs der spanischen Barockscholastik um die Legitimität der Conquista, der Eroberung Amerikas in der ersten Hälfte des 16. Jh., zugrunde liegt. Hierzu werde ich auf Juan Ginés de Sepúlveda und Bartolomé de Las Casas eingehen.

\subsection{Bellum iustum}

Der bellum iustum-Topos lässt sich, wie bereits erwähnt, in zwei Fragen unterteilen: 1. Wann hat wer das Recht, in einen Krieg einzutreten (ius ad bellum)? und 2. Wie ist ein Krieg gerechterweise zu führen (ius in bello)?

Die erste Frage zum ius ad bellum zielt darauf ab, gerechte Kriegsgründe (causae iustae) zu definieren, die hinreichend für den Kriegseintritt sind. Ferner soll bestimmt werden, wer zur Kriegsführung berechtigt ist, wer also die auctoritas legitima darstellt. Die zweite Frage des ius in bello beschäftigt sich mit der Art und Weise der Kriegsführung. Hier werden unter dem Begriff des debitus modus das Diskriminations- und das Proportionalitätsprinzip eingeführt.

Augustinus reflektiert über die Frage, ob auch Christen an einem Krieg teilnehmen dürfen und kommt zu einer bejahenden Antwort für den Fall, dass mit dem Krieg die Friedensordnung wiederhergestellt wird, die auf der von Gott gesetzten Schöpfungsordnung beruht (Hertz 1982, 430). Die Aufforderung Jesu zum radikalen Gewaltverzicht in der so genannten Bergpredigt (Mt 5, 38 ff.) relativiert er dahingehend, dass er sie nicht auf konkrete Handlungen bezieht, sondern auf die innere Bereitschaft des Menschen, die praeparatio cordis (Haltung des Herzens). Die Voraussetzung eines gerechten Krieges ist also immer die Verfehlung des anderen, denn „nur die Ungerechtigkeit der Gegenpartei nötigt dem Weisen gerechte Kriege auf" 
Ausgabe 2006

Jahrgang 3 Ausgabe 1

Schwerpunkt "Krieg und

Bildung" (hrsg. von

Elisabeth Zwick)

ISSN 1860-8213

\section{bildungsforschung \\ http://bildungsforschung.org}

(Augustinus 1985 [427], XIX, c. 7).

Dabei ist Krieg, so gerecht er auch sein mag, für Augustinus ein Übel, auf das nur „nach Ausschöpfung aller friedlichen Mittel“ (Budzik 1988, 300) als ultima ratio zurückgegriffen werden darf. Kernelement des bellum-iustumTopos bei Augustinus ist die Funktion des Krieges im Sinne einer iustitia vindicativa (strafende Gerechtigkeit). Hier erreicht Augustinus den Brückenschlag zum evangelikalen Auftrag Christi: Durch Krieg trägt man indirekt zur Feindesliebe bei, denn die ungerechte Gegnerschaft wird durch den Krieg wieder auf Gott hin orientiert. Ließe man den Ungerechten gewähren, entfernte er sich in dem Irrglauben, seine Ungerechtigkeiten seien lohnenswert, mehr und mehr von Gott und wäre so - eschatologisch verstanden - einer viel grausameren Strafe ausgesetzt als diese ihn im Krieg erwartet.

In Augustinus' Konzept setzt die recta intentio (rechte Absicht) der Art und Weise der Kriegsführung ihre Grenze. So sind nach Augustinus nur Mittel erlaubt, die zur Umkehr des Sünders beitragen, nicht aber solche, die Rachegelüste befriedigen oder von Gier, Grausamkeit und Unversöhnlichkeit zeugen (Augustinus 1891 [um 412], 672. Das Gewaltmonopol für den Fall eines gerechten Krieges liegt immer bei der staatlichen Autorität (auctoritas belli) (Hertz1982, 432). Augustinus verweist in diesem Zusammenhang auf die Ableitung weltlicher Herrschaft von Gott nach Röm 13, 1 ff.

Thomas von Aquin fragt, ob Kriegführen immer Sünde sei. Unter drei Bedingungen sei diese Frage zu verneinen: Eine Autorisierung durch den bevollmächtigten Fürsten (auctoritas principis), ein gerechter Grund (causa iusta) und eine rechte Absicht (recta intentio) müssten gleichzeitig vorliegen, um von einem gerechten Krieg sprechen zu können (Thomas v. Aquin 1933 ff. [1273], II-II, 40, 1). Zu den drei Bedingungen tritt die des debitus modus, des geringsten Mittels, faktisch hinzu. Thomas nimmt Bezug auf das LukasEvangelium, in dem es heißt: „Auch die Soldaten fragten ihn: Was sollen denn wir tun? Und er sagte zu ihnen: Mißhandelt niemand, erpreßt niemand, begnügt euch mit eurem Sold!" (Lk 3, 14). Das biblisch-moraltheologische Prinzip wird so zum ius in bello.

Ausgehend von Augustinus und Thomas entfachte sich in der ersten Hälfte des 16. Jh. im Zusammenhang mit der Frage "Ist die Conquista ein bellum iustum"? eine Kontroverse zwischen den Kolonisten um Hofchronist Sepúlveda, welche die Conquista argumentativ unterstützten und den IndioVerteidigern um Las Casas, dem "Apostel der Indios“, die sich eine missionszentrierte Kolonialisierung mit friedlichen Mitteln vorstellten. 
Ausgabe 2006

Jahrgang 3 Ausgabe 1

Schwerpunkt "Krieg und

Bildung" (hrsg. von

Elisabeth Zwick)

ISSN 1860-8213

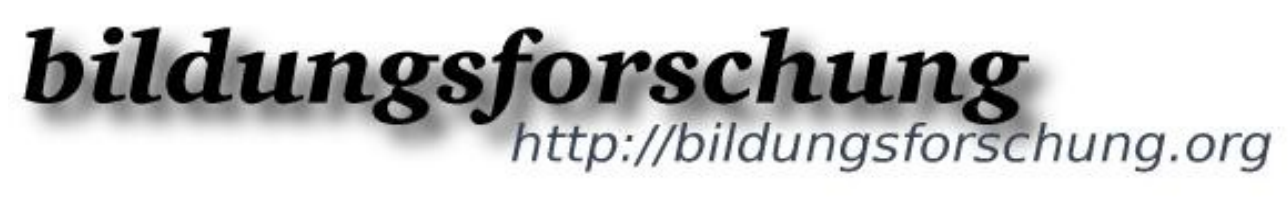

Hinsichtlich der Frage, wer das Recht zur Kriegsführung hat, sind sich Sepúlveda und Las Casas weitgehend einig: Allein der Fürst hat als auctoritas legitima das Recht, einen Krieg zu führen. Ausdrücklich bezieht Sepúlveda den obersten Kirchenfürsten, den Papst, mit ein, denn im Fall der Conquista habe Papst Alexander VI. von diesem Recht der auctoritas legitima Gebrauch gemacht, indem er den kastilischen Königen den Auftrag gab, die Indios zu unterwerfen.

Keine Einigkeit hingegen lässt sich bei der Frage nach den causae iustae der Conquista ausmachen. Für Sepúlveda gibt es der Tradition folgend grundsätzlich drei causae iustae für einen gerechten Krieg: Erstens die Verteidigung gegen einen ungerechten Angriff, zweitens die Rückgewinnung ungerecht einverleibter Kriegsbeute und drittens die Bestrafung eines bislang ungesühnten Rechtsbruchs (Sepúlveda 1951 [1545], 19). Wichtig für die gerechte Verteidigung sei dabei die Klassifizierung des Angriffs als ungerecht. Bei der Rückgewinnung ungerecht einverleibter Kriegsbeute bezieht sich Sepúlveda auf das Buch Genesis, in dem beschrieben wird, wie Abraham gegen den König von Elam Krieg führt (Gen 14), der zuvor Abrahams Neffen Lot, dessen Frauen und Besitz geraubt habe und erweitert das Recht zur Kriegsführung aus diesem zweiten Grunde auf die Rehabilitierung Verbündeter. Die Bestrafung eines Rechtsbruchs schließlich sei aus Gründen der Abschreckung geboten (Sepúlveda 1951 [1545], 17). Da im Fall der Conquista keiner der drei traditionell genannten gerechten Kriegsgründe verfängt, bemüht sich Sepúlveda um die Beschreibung weiterer causae iustae: Erstens die natürliche Inferiorität, zweitens die Sünden wider die Natur, drittens die Bewahrung Unschuldiger vor Anthropophagie und Opfertod sowie viertens die schnellere Verbreitung der christlichen Religion.

Im Zusammenhang mit der Interventionsthematik ist hier v. a. die Bewahrung Unschuldiger bedeutsam. Aus den Riten und Praktiken, die nach Sepúlveda der Natur zuwider laufen, entsteht eine Nothilfesituation, d. h. es geht nicht nur um die Bestrafung der Täter, sondern auch um den Schutz der (potentiellen) Opfer. Sepúlveda argumentiert sowohl theologisch-ethisch mit dersocietas (Gemeinschaft) des Menschengeschlechts, als auch biblisch mit dem Hilfeschrei aus dem Buch der Sprichwörter [3] und dem im LukasEvangelium verzeichneten Fürsorgegebot für den Nächsten, das sich im so genannten Gleichnis vom barmherzigen Samariter offenbart (Lk 10, 30 ff.).

Las Casas, der den Krieg nicht bloß als akademisches Problem betrachtet, sondern im Gegensatz zu Sepúlveda seine Kriegserlebnisse mit in die Analyse einbringt, weist die causae iustae der natürlichen Inferiorität der indigenen Bevölkerung, der Sünden wider die Natur sowie der schnelleren und 
Ausgabe 2006

Jahrgang 3 Ausgabe 1

Schwerpunkt "Krieg und

Bildung" (hrsg. von

Elisabeth Zwick)

ISSN 1860-8213

\section{bildungsforschung \\ http://bildungsforschung.org}

sichereren Verbreitung des Christentums zurück. Allein hinsichtlich des Aspekts "Schutz der Opfer" ist er prinzipiell mit Sepúlveda einig: die Befreiung Unschuldiger sei in der Tat grundsätzlich ein möglicherweise gerechter Kriegsgrund (Las Casas 1988 [1552], 360). Las Casas weist jedoch die Applikation dieser causa iusta auf den Krieg gegen die Indios zurück, da zum einen nur einige wenige Völker Amerikas von der Notwendigkeit zum Opferschutz betroffen seien und zum anderen ein Befreiungskrieg gegen die Indios seinen gerechten Grund konterkarieren würde, wenn hinsichtlich des ius in bello keine Gerechtigkeit der Kriegsführung $\mathrm{zu}$ erzielen sei (Gillner 1997, 233). Im Gegenteil: Er hielt unter den gegebenen Umständen der Conquista einen Krieg der Indios gegen die Spanier für gerecht und behautet, dass jene „verpflichtet [sind], gegen diese zu kämpfen, sie zu töten, sie festzunehmen und alle Rechte auszuüben, die aus einem gerechten Krieg in Übereinstimmung mit dem Völkerrecht folgen." (Las Casas 1988 [1552], 460).

Der debitus modus (gebotenes Maß) des gerechten Krieges liegt nach Sepúlveda in der Mäßigung hinsichtlich der Mittel. Mit Augustinus verurteilt er die möglichen Auswüchse eines Krieges in Racheakten und willkürlichen Grausamkeiten. Statt dessen gemahnt er zur Einhaltung des Diskriminationsund des Proportionalitätsprinzips. Beim Diskriminationsprinzip geht es darum, Unschuldige zu schonen, wobei er damit nicht wie das moderne Kriegsrecht zwischen Soldaten des Gegners und Zivilisten unterscheidet, sondern zur Schonung von Botschaftern, Ausländern und Klerikern sowie sakralen Gegenständen [4] aufruft. Die Einhaltung des Proportionalitätsprinzips wird hier nicht nur für die Kampfhandlungen, sondern auch für das Verhalten gegenüber dem Feind nach Beendigung des Krieges gefordert. [5] Übertragen auf die Conquista und den Kriegsgrund "Opferschutz" bedeutet das, die zu erwartende Zahl der Kriegsopfer solle kleiner sein als die Zahl zukünftiger Opfer naturrechtswidriger Praktiken. Das Proportionalitätsprinzip bleibe, so Sepúlveda, gewahrt, da bei einer jährlichen Opferzahl von 20.000 Menschen jeder noch so blutige Krieg gerechtfertigt sei (Sepúlveda 1951 [1545], S. 61).

Wie Sepúlveda geht auch Las Casas grundsätzlich davon aus, dass die Zahl der Opfer eines Krieges geringer sein muss als die Zahl der potentiellen Opfer des Vorkriegszustands, nur kommt er im Fall der indianischen Völker auf eine völlig andere Zahl: Er spricht von „nicht einmal 50“ (Las Casas 1988 [1552], 368 f.).

Ich möchte nun die wichtigsten Begriffe des bellum iustum-Topos auf Interventionskriege in unserer Zeit beziehen und einen aktuellen Vorschlag zur Verrechtlichung des "humanitären Interventionismus“ anhand der historischen, aber nicht veralteten Kriterien zum ius ad bellum und ius in 
Ausgabe 2006

Jahrgang 3 Ausgabe 1

Schwerpunkt "Krieg und

Bildung" (hrsg. von

Elisabeth Zwick)

ISSN 1860-8213

\section{bildungsforschung \\ http://bildungsforschung.org}

bello prüfen. Es handelt sich um einen Vorschlag aus dem Jahre 2001, um das Gutachten The Responsibility To Protect der International Commission on Intervention and State Sovereignty (ICISS). Die Frage ist, ob das, was dort vorgeschlagen wird, vor dem Hintergrund des historischen bellum iustumTopos tatsächlich geeignet ist, zur Grundlage einer Normierung des just war, des „gerechten Interventionskriegs“ gemacht zu werden? Hält der Vorschlag einer kritischen Reflexion auf die lascasianischen Kriterien des bellum iustum stand? Ich möchte insbesondere diese betrachten, da sie in ihrem friedensethischen Duktus am besten zur Richtschnur eines Interventionsrechts taugen.

\subsection{Just war}

In Anlehnung an die historische recta intentio fasst der Bericht die „right intention“ in folgendem Leitsatz zusammen: „The primary purpose of the intervention, whatever other motives intervening states may have, must be to halt or avert human suffering. Right intention is better assured with multilateral operations, clearly supported by regional opinion and the victims concerned." (IDRC 2001, XII). Der Schutz Unschuldiger - bei Las Casas einziger Interventionsgrund - wird im Kommissionsbericht zum "wichtigsten“ Grund. Gefährlich und ganz unlascasianisch ist der Zusatz „whatever other motives intervening states may have", hatte Las Casas doch in klaren Worten definiert, was eine Intervention auszeichnet: Unter den strengen Grenzen des ius in bello darf nur zum Schutz Unschuldiger interveniert werden. Die causa iusta des ius ad bellum ist freizuhalten von geostrategischen und ideologischen Partikularinteressen - und seien diese noch so löblich, wie etwa seinerzeit die vom Antipoden Sepúlveda vorgebrachte „schnelle Missionierung“.

Nur für den Fall von „serious and irreparable harm occurring to human beings, or imminently likely to occur“, einhergehend mit „large scale loss of life, actual or apprehended, with genocidal intent or not" sind militärische Interventionen gerechtfertigt (ebd.). Dann allerdings folgt aus diesem „just cause" - und diese Implikation erinnert an Las Casas' pathetische Rede von der "völkerrechtlichen Pflicht" der Indios, sich gegen die spanischen Aggressoren zur Wehr zu setzen - die Pflicht zum Eingriff mit sich, die sich gegen geltendes Völkerrecht - über Souveränität, Nichteinmischung und Gewaltverbot erhebt: "The principle of non-intervention yields to the international responsibility to protect.“ (IDRC 2001, XI). Von „responsibility“ (Verantwortung) ist die Rede, nicht etwa von „possibility“ (Möglichkeit). In der Praxis kann daraus nur eine "duty“, eine Pflicht, werden, die eigentlich dem 
Ausgabe 2006

Jahrgang 3 Ausgabe 1

Schwerpunkt "Krieg und

Bildung" (hrsg. von

Elisabeth Zwick)

ISSN 1860-8213

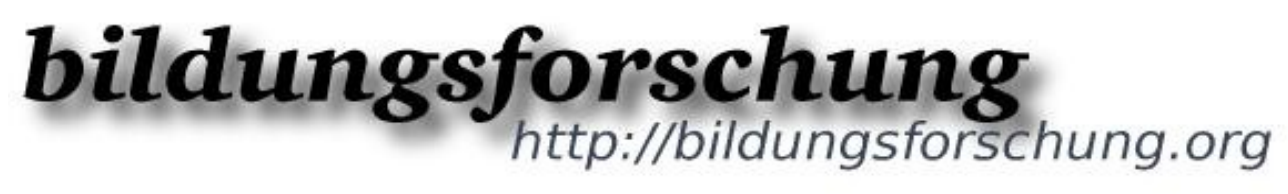

Staat selbst obliegt - als Träger der Gewalt auf seinem Hoheitsgebiet -, in Fällen des Staatsversagens aber auf die Weltgemeinschaft übergeht. Grundsätzlich bleibt also die Zuständigkeit und Verantwortung beim Staat. Erst wenn sich zeigt, dass dieser nicht in der Lage oder nicht willens ist, dieser Verantwortung gerecht $\mathrm{zu}$ werden, ist die Weltgemeinschaft am Zug. Entscheidend ist der Wandel des Souveränitätsbegriffs, der dem zugrunde liegt: Wenn überhaupt noch von staatlicher Souveränität gesprochen werden kann, dann nur im Sinne von Verantwortung für die Menschen.

Doch wer kann im Auftrag der Weltgemeinschaft diese Pflicht erfüllen? Wer kann unter den Bedingungen dieses Anspruchs heute die Autorität sein, die um im Tenor der Barockscholastik zu sprechen - im weltlichen Bereich keine höhere über sich hat? Wohl nur die Vereinten Nationen. Ihnen allein gebührt im 21. Jahrhundert das Kriegseintrittsrecht. Sie sind die legitima potestas („legitimate authority“), die allein kriegsführungsberechtigt ist. Auch hier kann zur ersten Orientierung auf das Gutachten der ICISS zurückgegriffen werden, das in der Frage nach der zum Krieg rechtmäßig autorisierenden Instanz eindeutig Stellung bezieht: „The UN, whatever arguments may persist about the meaning and scope of various Charter provisions, is unquestionably the principal institution for building, consolidating and using the authority of the international community.“ (IDRC 2001, 48). Konkret bedeutet das: „There is no better or more appropriate body than the United Nations Security Council to authorize military intervention for human protection purposes." (IDRC 2001, XII). Dabei muss es allerdings auch um die globale Anerkennung einer UN-Ethik gehen, d. h. um „die Bereitschaft, die UN-Autorität nicht für eine Durchsetzung illegitimer Interessen zu instrumentalisieren und [...] an der Verrechtlichung der internationalen Beziehungen mitzuwirken " (Beestermöller 2003, S. 83).

Auch unter einem UN-Mandat dürfen Interventionen nur dann in Angriff genommen werden, wenn sie das äußerste Mittel, also die ultima ratio (,last resort“) darstellen. Die ICISS schreibt dazu: „Military intervention can only be justified when every non-military option for the prevention or peaceful resolution of the crisis has been explored, with reasonable grounds for believing lesser measures would not have succeeded." (IDRC 2001, XII). Hier wird noch einmal deutlich, das nicht alles zuvor durchexerziert werden muss, sondern die Erfolgsprognostik zum Zeitpunkt der Erwägung entscheidet, ob sich die nicht-militärische, also diplomatische, politische oder wirtschaftliche Möglichkeit zu ergreifen lohnt. Das Handeln in Notsituationen hat nicht endlos Zeit, irgendwann müssen Maßnahmen ergriffen werden.

Sind diese militärischer Art, dann tritt für die schwierige Frage des Wie (ius 
Ausgabe 2006

Jahrgang 3 Ausgabe 1

Schwerpunkt "Krieg und

Bildung" (hrsg. von

Elisabeth Zwick)

ISSN 1860-8213

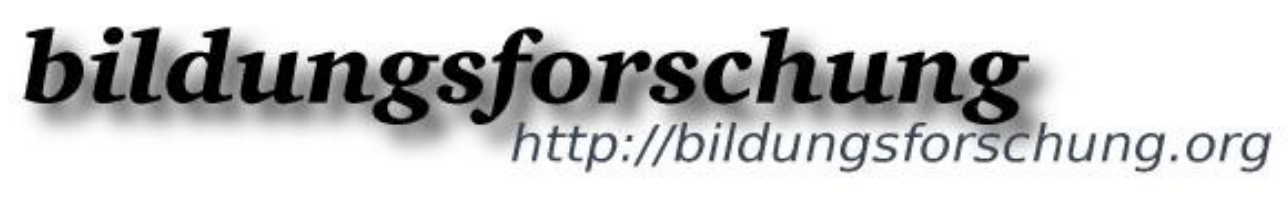

in bello) die Problematik des debitus modus auf. Eine noch so gerechte Begründung wird zur Farce, wenn der Kriegsverlauf Gräuel hervorruft, die selbst das Maß an Menschenrechtsverletzungen übersteigen, das einst die Intervention nötig machte. Las Casas sieht diese Gräuel mit eigenen Augen und verweigert der Conquista infolgedessen das Attribut „iusta“. Wie Las Casas verlangt auch die ICISS Proportionalität, die sich ausdrückt in „proportional means" („The scale, duration and intensity of the planned military intervention should be the minimum necessary to secure the defined human protection objective.", ebd.) und „reasonable prospects“ („There must be a reasonable chance of success in halting or averting the suffering which has justified the intervention, with the consequences of action not likely to be worse than the consequences of inaction." ebd.). Die Entscheidung, ob Proportionalität gewährleistet ist, sollte - auch das ist ganz im lascasianischen Sinne - unter Einbeziehung von NROs geschehen, die vor Ort sind und bei der Beurteilung auf empirische Daten zurückgreifen können, die den Entscheidungsträgern im Sicherheitsrat fehlen. Insoweit ist es nur konsequent, wenn die Kommission unter ihren „operational principles“ u. a. eine "maximum possible coordination with humanitarian organizations" (IDRC 2001, XIII) fordert. Wären die Erfahrungen des Las Casas und seiner Gesinnungsfreunde früher in den Diskurs um die Legitimität der Conquista eingeflossen, hätten also die Entscheidungsträger des Indienrats im fernen Spanien gewusst, was wirklich geschieht, hätten $u$. U. viele Menschenrechtsverletzungen in Lateinamerika verhindert werden können. [6]

Die Verantwortung der Weltgemeinschaft ist jedoch in den Augen der ICISS nicht nur eine reaktiv-militärische („responsibility to react“), sondern eine weitergehende. Sie erstreckt sich auf die Prävention („responsibility to prevent“) und den Wiederaufbau nach dem Krieg („responsibility to rebuild“), was eine ganz entscheidende Erweiterung der Interventionskonzeption darstellt und im Einklang mit Las Casas' christlicher Friedensorientierung steht. Gerade die Präventionsarbeit, die „sanfte, liebevolle, verständige Mission", die stets das Wohl des Anderen im Auge hat und nur durch Überzeugung eigene Werte zu vermitteln versucht, nicht mit Gewalt.

Dennoch kann es Fälle geben, in denen Menschenrechtsverletzungen ein Maß angenommen haben, dem es gewaltsam zu begegnen gilt. Las Casas betrachtet die Conquista selbst als eine solche, wenn er die Indios in die Pflicht nimmt, sich gewaltsam gegen die Spanier zur Wehr zu setzen (Las Casas 1988 [1552], 460). [7] Das Prinzip der Souveränität und Nichteinmischung gilt - insoweit trifft Las Casas den Kern des Art. 2, 7 der UN-Charta - solange, bis Interventionen - „die Anwendung von Zwangsmaßnahmen“ wie es im Text der Charta heißt - gerechtfertigt sind. 
Ausgabe 2006

Jahrgang 3 Ausgabe 1

Schwerpunkt "Krieg und

Bildung" (hrsg. von

Elisabeth Zwick)

ISSN 1860-8213

\section{bildungsforschung \\ http://bildungsforschung.org}

Gerechtfertigt sind sie bei Las Casas dann, wenn es einen gerechten Grund gibt, wobei Las Casas im Rahmen des bellum iustum-Topos höhere Maßstäbe anlegt als seine Zeitgenossen, Maßstäbe, die uns auch heute noch leiten können.

Doch wichtiger als militärische Zwangsmaßnahmen zum Zweck der Missionierung mit christlichen (damals) oder zivilreligiösen (heute) Glaubensinhalten, ist die Überzeugung des Anderen vom Nutzen dieses „Glaubens", ein Vorgang, der nur in der respektvollen Begegnung und durch eine Vermittlung, die den Anderen in seiner Andersartigkeit achtet, vollzogen werden. Jene „behutsame, liebevolle Mission“, die Las Casas gegen die "Schwertmission“ seiner Zeitgenossen setzt, kann auch heute im Zusammenhang mit der Vermittlung der Menschenrechte ein Leitbild sein. Auch heute wird wohl Bildung und Erziehung mehr zur Verwirklichung und Gewährleistung der Menschenrechte beitragen als ein „Menschenrechtskrieg“. Zur Prävention durch Menschenrechtserziehung möchte ich nun einige Ausführungen machen.

\section{Menschenrechtserziehung}

\subsection{Voraussetzungen}

Ausgangspunkt ist die Feststellung, dass die Menschenrechte auch das Recht auf die eigene Kultur umfassen, das Recht auf Anerkennung durch den Anderen als ebenbürtiges Völkerrechtssubjekt. Wird dieses Recht verletzt, entsteht für Las Casas schon qua lex naturalis ein Widerstandsrecht analog zu dem gegen die „innerstaatliche“ Tyrannei. Mit Blick auf die Indios billigt er ihnen das Recht zu, „ihren Götterkult und ihre Religion zu verteidigen und mit ihren bewaffneten Kräften gegen all jene auszurücken, die versuchen, sie ihres Kultes oder Religion zu berauben [...]“ (ebd.).

Übertragen auf die „zweite Globalisierung“ in unserer Zeit bedeutet dies, dass die Kommunitäten ihre kulturelle Identität $\mathrm{zu}$ verteidigen und sich gegen Uniformierung von außen $\mathrm{zu}$ wehren berechtigt sind: ob mit „bewaffneten Kräften" sei bestritten, denn auch Las Casas argumentiert hier nicht kohärent. Er stellt dem abendländischen Eurozentrismus des Kolonialzeitalters jedoch einen Pluralismus gegenüber, der auch heute ein Muster globaler Beziehungskultur sein sollte. Dabei ist jedoch die Frage, wie weit diese tolerante Haltung dem Anderen gegenüber gehen darf, wo die Grenze zum Relativismus überschritten wird. Las Casas Formel ist die, bei Menschenrechtsverletzungen die Tat, nicht den Täter zu bestrafen, ganz im 
Ausgabe 2006

Jahrgang 3 Ausgabe 1

Schwerpunkt "Krieg und

Bildung" (hrsg. von

Elisabeth Zwick)

ISSN 1860-8213

\section{bildungsforschung \\ http://bildungsforschung.org}

Sinne der christlichen Feindesliebe als „Entfeindungsliebe“, die den Irrtum und die Sünde bekämpft, den Irrenden und Sünder aber verschont. Vor dem Hintergrund kultureller und religiöser Eigenheit der autochtonen Bevölkerung betrachtet Las Casas die "Menschenrechtsverletzungen“ in den indianischen Gemeinschaften als entschuldbare Irrtümer (Las Casas 1988 [1552], 422), die es durch Aufklärung zu bekämpfen gilt, nur im äußersten Fall mit Gewalt. Hinsichtlich des Aspekts „Schutz der Opfer" verfolgt er dann, kurz gesagt, die Strategie Befreiung ja, Bestrafung nein. Nur so kann aus dem Zustand des Waffenstillstands, der Ziel einer gewaltsamen Intervention ist, wirklicher Friede entstehen. In diesem Sinne ist heute zu fordern, der Verletzung universalistischer Prinzipien, also liberaler oder sozialer Menschenrechte des Individuums, zunächst mit friedlichen, also politischen und wirtschaftlichen Mitteln entgegenzutreten, ehe an militärische Intervention gedacht wird, wobei diese entsprechende Nachbereitung erfordern, denn Krieg kann nur die nötigen Mindestvoraussetzung für ein erfolgreiches rebuilding schaffen, Krieg stiftet jedoch keinen Frieden. [8]

Daran schließt sich unmittelbar der Schutz vor blindem Sendungsbewusstsein an. Denn kein Grund für Interventionen darf in Las Casas Augen religiöser (heute: politischer) Missionseifer sein. Zwar ist es wünschenswert, dass alle Menschen unter dem Dach der christlichen Kirche (heute: in einer Demokratie) leben, jedoch darf zur Erreichung dieses Ziels kein Blut vergossen werden, denn gewaltsam installierter Ritus (heute: gewaltsam installiertes Rechtssystem) spottet der zugrundeliegenden Intention. Dies beschreibt er im Hinblick auf die Evangelisation sehr nachdrücklich. Übertragen bedeutet dies, dass Kernaspekte des $\mathrm{zu}$ vermittelnden Wertekanons niemals auf dem Wege der Vermittlung konterkariert werden dürfen. Wenn Las Casas im Hinblick auf die Schwertmission fragt: „Was hat die Gute Nachricht mit den Verletzungen, Gefangenschaften, Massensterben, Bränden, Zerstörungen der Städte und dem allgemeinen Übel des Krieges zu tun?“ (Las Casas 1988 [1552], 504), so müssen wir heute fragen: „Was hat Demokratisierung und die Installation von Menschenrechten mit Folter und dem respektlosen Umgang mit religiösen oder kulturellen Symbolen zu tun?“. Und wenn die Kernfrage damals lautete: „Nützt der Eingriff der Errettung von Seelen oder schadet er ihr?“, so lautet sie heute: „Nützt der Eingriff der Verwirklichung der Menschenrechte oder schadet er ihr?" Interessant ist, dass auch Beck die Parallele zwischen Missionierung und Demokratisierung kolportiert. Er führt aus, dass „Menschenrechtskriege“ (2004, 212) zur Gewährleistungen eines globalen „Menschenrechtsregimes“ (213) einerseits „neue Hoffnung und Handlungsmöglichkeiten eröffne[n]“ (ebd.), zugleich aber die Gefahr beinhalten, dass die „irdische Religion der Menschenrechte“ (ebd.) 
Ausgabe 2006

Jahrgang 3 Ausgabe 1

Schwerpunkt "Krieg und

Bildung" (hrsg. von

Elisabeth Zwick)

ISSN 1860-8213

\section{bildungsforschung \\ http://bildungsforschung.org}

$\mathrm{zu}$ „Menschenrechts-Kreuzzüge[n]“ (215) führe.

Hier kommt einer sanften, einfühlsamen Evangelisation, wie sie Las Casas vorschwebt, eine zivile Menschenrechts- und Demokratieerziehung gleich, wie sie in jüngster Zeit immer wieder als unabdingbar thematisiert wird. [9]

Die Prinzipien einer friedensorientierten Menschenrechtserziehung sind dabei eine strikte Folgenorientierung, eine Dialogorientierung, die zur Argumentation befähigt und Gewalt als fortschrittshemmend ablehnt, [10] eine Komplexitätsorientierung, die Probleme nicht verkürzt, sondern deutlich offen legt sowie das Kontrovers-, das Öffentlichkeits- und das Explikationsprinzip, die noch einmal auf die Bedeutung des Arguments als kontrafaktisches Moment zur Gewalt verweisen (vgl. Birckenbach 1994, 159 ff.). Das Ziel der Menschenrechtserziehung ist selbstverständlich die Bildung einer neuen Generation, die Menschenrechte achtet und schützt und sensibel auf ihre Verletzung reagiert - möglichst im Rahmen friedlicher Methoden.

\subsection{Völkerrechtliche Grundlagen}

Nun komme ich - bevor ich die beiden Phasen der UNO-Arbeit darstellen werde - zu den völkerrechtlichen Grundlagen der Menschenrechtserziehung. Bereits die 1948 verkündete Allgemeine Erklärung der Menschenrechte (AEMR) enthält an prominenter Stelle, nämlich schon in der Präambel, eine interessante Anmerkung. Es wird darauf verwiesen, dass die Achtung der in der Erklärung verbrieften Rechte durch „Unterricht und Erziehung“ zu fördern sind (Präambel Abs. 5 AEMR). In Artikel 26 wird die Zielsetzung der schulischen und beruflichen Ausbildung bestimmt als die „volle Entfaltung der menschlichen Persönlichkeit und die Stärkung der Achtung der Menschenrechte und Grundfreiheiten" (Art. 26 Zf. 2 Satz l AEMR). Wir finden also schon bei den Menschenrechten selbst den Hinweis auf die Bedeutung von Bildung und Erziehung für die Verwirklichung dieser Rechte. Daher liegt es nahe, von einem "Menschenrecht auf Menschenrechtserziehung“ zu schließen.

Dieser Eindruck wird bestärkt, wenn man sich weitere Dokumente des Individualvölkerrechts anschaut. Im Internationalen Pakt über wirtschaftliche, soziale und kulturelle Rechte (1966) wird Bildung als Menschenrecht hervorgehoben und betont, dass „die Bildung auf die volle Entfaltung der menschlichen Persönlichkeit und des Bewusstseins ihrer Würde gerichtet sein und die Achtung vor den Menschenrechten und Grundfreiheiten stärken soll“ (Art. 13 Satz 1). Auch das Internationalen Übereinkommen zur Beseitigung jeder Form von Rassendiskriminierung aus dem gleichen Jahr weist auf den 
Ausgabe 2006

Jahrgang 3 Ausgabe 1

Schwerpunkt "Krieg und

Bildung" (hrsg. von

Elisabeth Zwick)

ISSN 1860-8213

\section{bildungsforschung \\ http://bildungsforschung.org}

Zusammenhang zwischen Erziehung und Menschenrechtsschutz hin, wenn die Vertragsstaaten verpflichtet werden, „unmittelbare und wirksame Maßnahmen, insbesondere auf dem Gebiet des Unterrichts, der Erziehung, Kultur und Information $\mathrm{zu}$ treffen, um Vorurteile $\mathrm{zu}$ bekämpfen, die $\mathrm{zu}$ Rassendiskriminierung führen, zwischen den Völkern und Rassen- oder Volksgruppen Verständnis, Duldsamkeit und Freundschaft zu fördern sowie die Ziele und Grundsätze der Charta der Vereinten Nationen, der Allgemeinen Erklärung der Menschenrechte, der Erklärung der Vereinten Nationen über die Beseitigung jeder Form von Rassendiskriminierung und dieses Übereinkommens zu verbreiten." (Art. 7). Schließlich wird im Internationalen Übereinkommen über die Rechte des Kindes (1989) der Zusammenhang zwischen Bildung und Achtung vor den Menschenrechten genannt (Art. 29). Kurzum: Wo immer auf völkerrechtlicher Ebene von Menschenrechten gesprochen wird, da wird zugleich die Menschenrechtserziehung erwähnt und dort, wo es um Bildung geht, wird die Achtung vor den Menschenrechten als wichtigster Inhalt genannt.

Nun ist es so, dass die Menschenrechte selbst nicht ganz unkritisch betrachtet werden. Die einen kritisieren die fehlende juristische Durchschlagskraft, andere das zu sehr auf das Individuum orientierte Rechtsverständnis, das als Erbe eines aus dem christlichen Gottebenbildlichkeitsgedanken entlehnten Menschenbildes ausschließlich auf die Autonomie und auf die individuelle Freiheit des Menschen fokussiere und in dieser Ausprägung in vielen gemeinschaftlich organisierten Stammeskulturen unbekannt sei. Diese okzidentozentrische Anthropologie christlich-abendländischer Provenienz könne auf diese Kulturen keine Anwendung finden. Wieder andere betonen, dieser Freiheits- und Autonomieduktus der Menschenrechte in ihrer vom „Westen“ proklamierten Form hemme den Entwicklungsprozess in der so genannten „Dritten Welt“. Die Entwicklungsländer Afrikas und Lateinamerikas könnten sich nicht alle Freiheitsrechte leisten, diese somit auch nicht gewährleisten. Wirtschaftliche Entwicklung habe Vorrang vor einer strikten Einhaltung der Menschenrechte. Im egalitaristischen Gerechtigkeitsverständnis sind soziale Sicherheit und tendentielle Gleichheit wichtiger als die freie Entfaltung des Einzelnen auf wirtschaftlicher, künstlerischer, politischer und wissenschaftlicher Ebene.

Ich möchte diese Einwände nicht diskutieren, denke aber, dass es Sinn hat, am Universalitätsanspruch der Menschenrechte festzuhalten, auch wenn de facto kulturpluralistische Gegebenheiten einer weltweiten Geltung der Menschenrechte im Wege stehen und eine Uniformierung - etwa im Wege eines militärischen Interventionismus als Dauerinstanz - wenig sinnvoll erscheint. Wichtig hingegen erscheint mir eine erzieherische Vermittlung in 
Ausgabe 2006

Jahrgang 3 Ausgabe 1

Schwerpunkt "Krieg und

Bildung" (hrsg. von

Elisabeth Zwick)

ISSN 1860-8213

\section{bildungsforschung \\ http://bildungsforschung.org}

Zeiten von zunehmender globaler Unübersichtlichkeit, wie ich sie in der Einleitung geschildert habe. Die UNO wollte und will dieser Notwendigkeit nachkommen. Sie forderte im Frühjahr 1993 in Montreal einen „Weltaktionsplan zur Erziehung zu Menschenrechten und Demokratie“ (zit. nach EPU et al 1997, 81 ff.), der im Schlussdokument der Weltkonferenz über Menschenrechte im Juni desselben Jahres in Wien aufgegriffen wurde. In einer Konkretisierung der Forderung verlangt es von der UNO, „eine Dekade der Vereinten Nationen für Menschenrechtserziehung mit dem Ziel der Förderung, Ermutigung und Bündelung dieser Aufklärungsmaßnahmen auszurufen" (Wiener Erklärung 1993, D 518).

1995 begann diese Dekade für Menschenrechtserziehung, die sich von 2005 bis 2015 im Weltprogramm für Menschenrechtsbildung fortsetzen soll. Die Ziele, Prinzipien und Ergebnisse dieser „Aktionen“ sollen im folgenden Abschnitt dargestellt werden.

\subsection{Dekade für Menschenrechtserziehung (1995-2004)}

Für die am 23. Dezember 1993 von der Generalversammlung einstimmig proklamierten Dekade für Menschenrechtserziehung wurde folgende Zielsetzung beschlossenen: Es gehe in den kommenden zehn Jahren grundsätzlich darum, durch die Vermittlung von Wissen und Fähigkeiten eine „Kultur der Menschenrechte“ zu stärken und dabei die Denkweise der Menschen auf die Achtung der Menschenrechte und Grundfreiheiten sowie auf die volle Entfaltung der Persönlichkeit und die Würde des Menschen zu orientieren (UN-Doc. A/51/506/Add. 1). Hier knüpft das praktische Programm an die völkerrechtlichen Normen an.

Doch wie kann und soll Menschenrechtserziehung einen Beitrag zur Erreichung dieser hehren Ziele leisten? Zwei Jahre nach dem Beschluss des Aktionsprogramms werden im Bericht über die Implementierung desselben Prinzipien einer effizienten Menschenrechtserziehung genannt (UN-Doc. A/51/506/Add. 1, Annex 1996, Zf. 9), die sich zu fünf Punkten zusammenfassen lassen: 1. Das Rechts-, Norm- und Wertverständnis, das der AEMR und den Pakten zugrunde liegt, soll geschärft werden, 2. Ein holistisches Verständnis der Menschenrechtsidee, das liberale und soziale Rechte zusammen betrachtet, soll gefördert werden, 3. Menschenrechtsbildung soll grundsätzlich alle Menschen erreichen können, unabhängig vom Alter, Geschlecht, Rasse, Religionszugehörigkeit oder gesellschaftlicher Stellung, wobei stets darauf $\mathrm{zu}$ achten ist, dass die Erziehung ohne Stereotype und Klischees erfolgt bzw. diese bekämpft, 4. Der Praxisbezug sollte gewährleistet sein, so dass die Menschen befähigt werden, konkrete Situationen ihrer 
Ausgabe 2006

Jahrgang 3 Ausgabe 1

Schwerpunkt "Krieg und

Bildung" (hrsg. von

Elisabeth Zwick)

ISSN 1860-8213

\section{bildungsforschung http://bildungsforschung.org}

Lebenswelt unter die abstrakten Werten und Normen zu subsumieren und 5. Die Interdependenz von Menschenrechten und Demokratie soll dadurch deutlich werden, dass bei der Vermittlung menschenrechtlicher Inhalte eine demokratische Form gewählt wird.

Bleibt die Frage, wer konkret angesprochen ist, aktiv zu werden und an wen sich die Programme richten. Als Verantwortliche für die Durchführung nennt das Aktionsprogramm zunächst die nationalen Regierungen und staatlichen Menschenrechtsinstitutionen, denen mit den Guidlines for national plans of action for human rights education (UN-Doc. A/52/469/Add. 1, Addendum 1997) Leitlinien zur Umsetzung der oben erwähnten Prinzipien und zur Durchführung nationaler Programme an die Hand gegeben wurden. Doch auch Nichtregierungsorganisationen sollen eingebunden werden. Adressaten sind insbesondere alle Menschen, die in ihrer beruflichen Tätigkeit menschenrechtliche Fragen behandeln (z. B. Juristen, Mitarbeiter in politischen Gremien und in der Verwaltung), mit menschenrechtsrelevanten Themen oft in Berührung kommen (z. B. Polizisten, Soldaten, Strafvollzugsbeamte, Entwicklungshelfer, Mitglieder von Nichtregierungsorganisationen) oder die als Multiplikatoren ihrerseits eine wichtige „Erziehungsfunktion“ tragen (Lehrer, Professoren, Journalisten, etc.). Hier bleibt im Aktionsplan offen, inwieweit bei den „Mitgliedern von Nichtregierungsorganisationen" auch Multiplikatoren in Religionsgemeinschaften angesprochen werden sollen, denn das Wort eines Predigers oder Imams hat häufig mehr Gewicht als ein Leitartikel oder eine Unterrichtsstunde zum Thema Menschenrechte. Die Exegese kanonischer Schriften im Lichte der Menschenrechte und die Betonung der Würde des Menschen, die in allen Religionen angelegt ist, stellt sicherlich einen entscheidenden Schlüssel zur Stärkung der Achtung der Menschenrechte dar. Umso wichtiger, dass sich Religionsgemeinschaften als „Nichtregierungsorganisationen“ aktiv in den Erziehungsprozess einbringen und hierzu auch ermutigt werden. Dieser Ansatz bleibt bei der UNO als säkularer Organisation leider unberücksichtigt.

Was wurde erreicht? Der Zwischenbericht aus dem Jahre 2000 (UN-Doc. A/55/360) hält fest, dass das Ausrufen der Dekade für Menschenrechtserziehung als Katalysator gewirkt habe. Es sei gelungen, staatliche und nicht-staatliche Organisation zusammenzuführen. Allerdings sei eine wachsende Notwendigkeit für eine erhöhte Koordination offenbar geworden. Auch sei das Potential an internationaler Zusammenarbeit im Bereich der Menschenrechtserziehung bei weitem nicht ausgeschöpft. Es bestehe schließlich nach wie vor eine Kluft zwischen Versprechungen und Verpflichtungen, die überbrückt werden müsse. 
Ausgabe 2006

Jahrgang 3 Ausgabe 1

Schwerpunkt "Krieg und

Bildung" (hrsg. von

Elisabeth Zwick)

ISSN 1860-8213

\section{bildungsforschung \\ http://bildungsforschung.org}

Das Hochkommissariat für Menschenrechte der Vereinten Nationen legte in den Jahren 2003 und 2004 weitere Berichte vor, die zu ähnlichen Ergebnissen kommen (UN-Doc. E/CN. 4/2004/93). Insbesondere wird noch einmal betont, dass die Zusammenarbeit zwischen den Vereinten Nationen, regionalen Staatenbünden und den Nationalstaaten einerseits sowie zwischen staatlichen und nicht-staatlichen Einrichtungen andererseits verbessert werden muss.

Zum Abschluss der Dekade verweist die Erklärung der Generalversammlung am 10. Dezember 2004 auf das Fazit der Berichte hin und kommt zu dem Ergebnis: Es war ein wichtiger Schritt, es bleibt aber noch viel zu tun. Das entscheidende sei das gestiegene Problembewusstsein: Menschenrechte stehen wieder ganz oben auf der Tagesordnung. Unbestritten sei es gelungen, die Bedeutung von Erziehung und Bildung als Katalysatoren nicht nur der liberalen Menschenrechte zu untermauern, sondern auch der sozialen, auf die gerade Entwicklungsländer verstärkt schauen. Bildung, Entwicklung und Menschenrechte werden vor diesem Hintergrund mehr und mehr als harmonischer Dreiklang empfunden, d. h. die elementare Verbindung von Armutsbekämpfung und Verbesserung der Bildungschancen, die nie ernsthaft bestritten wurde, wird um die Abhängigkeit der wirtschaftlichen Entwicklung von der Gewährleistung der Menschenrechte ergänzt und diese als Produkt auch und gerade von Bildung und Erziehung verstanden. Es setzt sich die Erkenntnis durch, dass Menschenrechte der wirtschaftlichen Entwicklung nicht im Weg stehen, sondern sie erst möglich machen. Bildung darf in diesem Zusammenhang nicht allein auf Sachgebiete bezogen erfolgen, sondern das Thema Menschenrechte einschließen.

Vom Hauptziel der Dekade für Menschenrechtserziehung, eine „Kultur der Menschenrechte" zu schaffen, ist die Welt - auch daran herrschte in der Generalversammlung Einmütigkeit - noch weit entfernt. Es wurde daher in Erwägung gezogen, das Aktionsprogramm um eine zweite Dekade zu verlängern. Dagegen wandten sich die USA und die EU gleichermaßen, denen eine andere Akzentuierung und eine verbindlichere Umsetzung des Programms vorschwebte, weg von den Versprechungen, hin zu möglichst konkreten Verpflichtungen und klaren Aufgabenzuweisungen.

Zum ersten Mal in ihrer Geschichte riefen die Vereinten Nationen daraufhin ein Weltprogramm für Menschenrechtsbildung aus (UN Press Release GA/1010317), dass sich durch eine höhere Verbindlichkeit auszeichnet und sich insoweit vom Programm der Dekade unterscheidet. Strikter ist das Weltprogramm bezüglich der Anforderungen - es gibt nun „Mindestanforderungen“, die jedes Mitgliedsland der UN erfüllen muss - und der Evaluierung, die nun nicht nur in Form eines Zwischenberichts, sondern 
Ausgabe 2006

Jahrgang 3 Ausgabe 1

Schwerpunkt "Krieg und

Bildung" (hrsg. von

Elisabeth Zwick)

ISSN 1860-8213

\section{bildungsforschung \\ http://bildungsforschung.org}

nach jeder „Phase“ erfolgen soll. Die erste Phase umfasst den Zeitraum 2005 bis 2007. Die konkreten Ziele der ersten Phase des Weltprogramms für Menschenrechtsbildung möchte ich abschließend kurz umreißen.

\subsection{Weltprogramm für Menschenrechtsbildung (2005- 2015): Erste Phase (2005-2007)}

Nachdem das Weltprogramm verabschiedet war, wurde über die Ziele und Schwerpunkte der ersten Phase verhandelt. Am 2. März 2005 legte UNGeneralsekretär Kofi Annan den überarbeiteten Aktionsplan des Weltprogramms vor (UN-Doc. A/59/525/Rev. 1). Im Mittelpunkt steht demnach die Menschenrechtserziehung in der Primar- und Sekundarschulbildung, die unter folgenden fünf Aspekten gestaltet werden soll: 1. Die Entwicklung einer partizipativen, menschenrechtsorientierten Bildungspolitik, die der Bildung der Lehrer und Ausbilder den Vorrang gibt, soll vorangetrieben werden, 2. Dazu sollen Fortbildungsmaßnahmen ergriffen werden, die geeignet sind, Lehrern und Ausbildern das menschenrechtliche Wissen, aber auch das nötige Verständnis und die Kompetenz zu vermitteln, 3. Die Realisierung und Implementierung bildungspolitischer Innovationen soll unter Beteiligung aller Akteure stattfinden, 4. An den Schulen soll ein solidarisches, an den Menschenrechten orientiertes Klima herrschen, in dem Schülerinnen und Schüler frei ihre Meinung sagen dürfen und an den Entscheidung demokratisch beteiligt werden und 5. Menschenrechtszentrierte Lehr- und Lernprozesse sollen durch die Entwicklung von entsprechenden Lehrplänen und Unterrichtsformen sowie die Bereitstellung geeigneter Mittel (insbesondere Lehrbücher) gefördert werden.

Jeder Mitgliedsstaat wird darüber hinaus verpflichtet, bis 2007 zumindest den Status quo der Menschenrechtsbildung im Schulsystem zu ermitteln, eine spezifische Implementierungs-strategie der Erziehungsziele zu erarbeiten und mit der Implementierung $\mathrm{zu}$ beginnen. Verantwortlich sind die jeweiligen nationalen Bildungsministerien. Es bleibt abzuwarten, ob sie dieser Pflicht nachkommen.

\section{Fazit}

Zum Schluss möchte ich kurz resümieren, dass beides nötig zu sein scheint, um Menschenrechte wirkungsvoll zu schützen und ihre weltweite Geltung zu gewährleisten: Reaktion und Prävention. Es kann Gründe geben, militärisch einzugreifen, um den Menschenrechtsschutz zu gewährleisten, etwa wenn ein Völkermord droht, es ist aber mindestens ebenso wichtig, im lascasianischen 
Ausgabe 2006

Jahrgang 3 Ausgabe 1

Schwerpunkt "Krieg und

Bildung" (hrsg. von

Elisabeth Zwick)

ISSN 1860-8213

\section{bildungsforschung \\ http://bildungsforschung.org}

Geist der „sanften Mission“ die Erziehung zum Frieden und zur Achtung der Menschenrechte zu befördern, damit genau diese Interventionsgründe nicht eintreten.

Nur das Zusammenwirken von reaktiven und präventiven Maßnahmen kann die Menschenrechtsidee weltweit befördern. Der ambitionierte Versuch, dieser Erkenntnis einen völkerrechtlichen Rahmen zu geben und die Vereinten Nationen als Träger beider Optionen im Sinne einer effizienten Durchführung derselben $\mathrm{zu}$ reformieren, ist deshalb $\mathrm{zu}$ begrüßen. Es ist wünschenswert, dass sowohl das Gutachten der ICISS, als auch die Programme zur Menschenrechtserziehung und die Evaluierungsberichte ernsthaft geprüft werden und in naher Zukunft zu geltenden Völkerrechtsnormen und damit zur dauerhaften Grundlage einer globalen Institutionenpraxis führen werden, welche die Ohnmacht einer handlungsunfähigen UNO im Fall von humanitären Interventionen ebenso der Vergangenheit angehören lässt wie das voluntaristische Stückwerk im Bereich präventiver Maßnahmen. Es ist dabei entscheidend, die Prioritäten nicht zu vertauschen. In den zu bildenden Normen muss klar zum Ausdruck kommen, dass die erzieherische Prävention den Regelfall darstellt, die militärische Reaktion hingegen den Ausnahmefall.

\section{Fußnoten}

[1]Clausewitz spricht in diesem Sinne von „Fortsetzung der Politik mit anderen Mitteln“ (1990, 179).zurück

[2]Mitglieder der Kommission waren Gareth Evans und Mohamed Sahnoun als Vorsitzende sowie Gisèle Côté-Harper, Lee Hamilton, Michael Ignatieff, Vladimir Lukin, Klaus Naumann, Cyril Ramaphosa, Fidel Ramos, Cornelio Sommaruga, Eduardo Stein und Ramesh Thakur als einfache Mitglieder.zurück

[3],BEefreie jene, die man zum Tod schleppt; die zur Hinrichtung wanken, rette sie doch!“ (Spr 24, 11).zurück

[4]Sakral selbstverständlich aus Sicht des christlichen Glaubens. Die Tempelanlagen und Heiligtümer der Indios wurden im Zuge des „heiligen Krieges" gegen den Blasphemie, Irrglauben und Götzendienst zerstört.zurück

[5]Interessanterweise erscheint hier erstmals die neutestamentliche lex nova der Liebe, die dem Feind nach Beendigung des Krieges entgegenzubringen sei.zurück

[6]Tatsächlich zeitigten die Eingaben und das persönliche Engagement des Las Casas am Hof Karls V. Erfolge. Die Verabschiedung der Leyes Nuevas 
Ausgabe 2006

Jahrgang 3 Ausgabe 1

Schwerpunkt "Krieg und

Bildung" (hrsg. von

Elisabeth Zwick)

ISSN 1860-8213

\section{bildungsforschung \\ http://bildungsforschung.org}

(1542) geht etwa auf lascasianische Interventionen zurück. Wenn diese neuen Gesetze auch viel zu spät erlassen wurden und auch nicht zu grundlegenden Änderungen in der Praxis der Kolonialverwaltung führten, waren sie doch ein erster Schritt in die richtige Richtung. Ohne die Arbeit des Dominikaners wären wohl die Missstände in Lateinamerika ganz am vielbeschäftigten Kaiser vorbeigegangen, stand der doch vor der gewaltigen Aufgabe, sein (europäisches) Reich politisch $\mathrm{zu}$ einen, territorial zusammen $\mathrm{zu}$ halten, militärisch vor den einfallenden Türken zu verteidigen sowie konfessionell wieder unter die päpstliche Oberherrschaft zu versammeln. Dabei musste er dann noch die Kredite an die deutschen Banken der Fugger und Welser zurück zahlen - da blieb für Kolonialethik wenig Zeit.zurück

[7]In diesem Zusammenhang rechtfertigt er auch den Übergriff auf den Conquistador Pedro de Alvarado und dessen Soldaten in Guatemala als "gerechte Verzweiflungstat" (Las Casas 1992 [1552], 55).zurück

[8]Vgl. Lutz (2001).zurück

[9]So in aktuellen Publikationen von Lenhart (2003) und Lohrenscheit (2004).zurück

[10]Zum Kontext von Argumentationskultur und Zivilisationsniveau vgl. Gil, der unter Rekurs auf Senghass' „Hexagon“ zeigt, wie Argumentationen auf den hohen soziokulturellen Entwicklungsstand, der gegeben sein muss, damit überhaupt erst ein öffentlicher Diskurs statt finden kann, stabilisierend wirken, indem sie die friedliche Konfliktlösung an die Stelle der Gewalt setzen (2005, 60 ff.). Dabei spielt das Erlernen von Argumentation als Technik der Auseinandersetzung eine besondere Rolle, der die attische Philosophie mit der $\Pi \alpha \iota \delta \varepsilon \imath \alpha$ gerecht zu werden versuchte (70 ff.), die durchaus als antikes Vorbild einer gegenwärtigen Menschenrechtserziehung gelten kann.zurück

\section{Autor}

Josef Bordat Dipl.-Ing., M.A., Doktorand (Philosophie)

Berlin

E-Mail: josef bordat@hotmail.com

\section{Literatur}

- Augustinus (1985). Vom Gottesstaat. München 1985.

- Augustinus (1891). Contra faustum. In: Corpus Scriptorum Ecclesiasticorum Latinorum 25. Prag. 
Ausgabe 2006

Jahrgang 3 Ausgabe 1

Schwerpunkt "Krieg und

Bildung" (hrsg. von

Elisabeth Zwick)

ISSN 1860-8213

\section{bildungsforschung \\ http://bildungsforschung.org}

- Bald, D. (1999). Die Intervention im Kovoso. Macht und Moral als Auftrag der neuen Bundeswehr. In: Vierteljahresschrift für Sicherheit und Frieden, Jg. 17, Nr. 2, S. 93 ff.

- Beck, U. (1999). Über den postnationalen Krieg. In: Blätter für deutsche und internationale Politik, Jg. 24, Nr. 8, S. 984 ff.

- Beck, U. (2004). Der kosmopolitische Blick oder: Krieg ist Frieden. Frankfurt a. M.

- Beestermöller, G. (2003). Krieg gegen den Irak - Rückkehr in die Anarchie der Staatenwelt? Ein kritischer Kommentar aus der Perspektive einer Kriegsächtungsethik. Stuttgart.

- Birckenbach, H.-M. (1994). Fact-Finding: Ein Instrument friedensfördernder Menschenrechts-politik. Erfahrungen aus dem Konflikt um die estnische und lettische Staatsbürgerschaft. In: Koch, J. I Mehl, R. (Hrsg.): Politik der Einmischung. Zwischen Konfliktprävention und Krisenintervention. Baden Baden, S. $143 \mathrm{ff}$.

- Budzik, S. (1988). Doctor pacis. Theologie des Friedens bei Augustinus. Innsbruck.

- Clausewitz, C. v. (1990). Vom Kriege. Bonn.

- Europäisches Universitätszentrum für Friedensstudien (EPU) et al. (1997). Erziehung für Frieden, Menschenrechte und Demokratie im UNESCO-Kontext. Stadtschlaining.

- Gil, T. (2005). Argumentationen. Der kontextbezogene Gebrauch von Argumenten. Berlin.

- Gillner, M. (1997). Bartolomé de Las Casas und die Eroberung des indianischen Kontinents. Das friedensethische Profil eines weltgeschichtlichen Umbruchs aus der Perspektive eines Anwalts der Unterdrückten. Stuttgart.

- Hegel, G. W. F. (1986). Grundlinien der Philosophie des Rechts oder Naturrecht und Staatswissenschaft im Grundrisse. In: Ders.: Werke. Bd. 7, Frankfurt a. M.

- Hertz, A. (1982). Die Lehre vom, gerechten Krieg' als ethischer Kompromiß. In: Ders. (Hrsg.): Handbuch der christlichen Ethik. Bd. 3, Freiburg i. Br., S. 425 ff.

- IDRC (2001). The Responsibility to Protect. Report of the International Commission on Intervention and State Sovereignty. Ottawa.

- Las Casas, B. d. (1988). Apología. In: Castañeda Delgado, P. (Hrsg.): Bartolomé de Las Casas. Obras completas. Bd. 9, Madrid.

- Las Casas, B. (1992). Tratados de 1552. Hrsg. v. Hernández, R. / Galmés L. In: Castañeda Delgado, P. (Hrsg.): Bartolomé de Las Casas. Obras completas. Bd. 10, Madrid.

- Lenhart, V. (2003). Pädagogik der Menschenrechte. Opladen. 
Ausgabe 2006

Jahrgang 3 Ausgabe 1

Schwerpunkt "Krieg und

Bildung" (hrsg. von

Elisabeth Zwick)

ISSN 1860-8213

\section{bildungsforschung \\ http://bildungsforschung.org}

- Lohrenscheit, C. (2004). Das Recht auf Menschenrechtsbildung. Grundlagen und Ansätze einer Pädagogik der Menschenrechte. Frankfurt a. M.

- Lutz, D. S. (2001). Vorwort. In: Ronnefeldt, C.: Die Neue NATO, Irak und Jugoslawien. Minden.

- Ross, J. (2001). Hegel der Bundesrepublik. Krieg ist in der Welt. Jürgen Habermas, der Philosoph der Konsensgesellschaft, erhält den Friedenspreis. Eine Würdigung aus gegebenem Anlass. In: Die Zeit. Jg. 56, Nr. 42.

- Sepúlveda, J. G. d. (1952). Democrates segundo o de las justas causas de la guerra contra los indios. Madrid.

- Thomas v. Aquin (1933). Deutsche Thomas-Ausgabe. Vollständige, ungekürzte deutsch-lateinische Ausgabe der Summa theologica. 36 Bde. Hrsg. v. d. Albertus-Magnus-Akademie Walberberg b. Köln, Heidelberg / München u. Graz / Wien / Salzburg 1933 ff.

\section{Zitierte Rechtsnormen und UN-Dokumente (chronologisch)}

- Allgemeine Erklärung der Menschenrechte (1948)

- Internationaler Pakt über wirtschaftliche, soziale und kulturelle Rechte (1966)

- Internationales Übereinkommen zur Beseitigung jeder Form von Rassendiskriminierung (1966)

- Internationales Übereinkommen über die Rechte des Kindes (1989)

- Schlussdokument der Weltkonferenz über Menschenrechte (1993): "Wiener Erklärung“.

- UN-Doc. A/51/506/Add. 1 (1994): „Plan of Action for the United Nations Decade for Human Rights Education, 1995-2004: Human rights education - lessons for life".

\section{Zitation}

Empfohlene Zitation: Bordat, Josef (2006). Menschenrechtskrieg und Menschenrechtserziehung. In: bildungsforschung, Jahrgang 3, Ausgabe 1, URL: http://www.bildungsforschung.org/Archiv/2006-01/menschenrechte/

[Bitte setzen Sie das Datum des Aufrufs der Seite in runden Klammern und verwenden Sie die Kapitelnummern zum Zitieren einzelner Passagen] 